\title{
Mid-IR emission of galaxies in the Virgo cluster and in the Coma supercluster
}

\section{The nature of the dust heating sources}

\author{
A. Boselli ${ }^{1}$, J. Lequeux ${ }^{2}$, and G. Gavazzi ${ }^{3}$ \\ ${ }^{1}$ Laboratoire d'Astrophysique de Marseille, BP 8, Traverse du Siphon, 13376 Marseille Cedex 12, France \\ e-mail: Alessandro.Boselli@oamp.fr \\ 2 Observatoire de Paris, LERMA, 61 Av. de l'Observatoire, 75014 Paris, France \\ e-mail: James . Lequeux@obspm.fr \\ 3 Universitá degli Studi di Milano-Bicocca, P.zza della Scienza 3, 20126 Milano, Italy \\ e-mail: Giuseppe.Gavazzi@mib.infn.it
}

Received 19 May 2004 / Accepted 27 July 2004

\begin{abstract}
We study the relationship between the mid-IR $(5-18 \mu \mathrm{m})$ emission of late-type galaxies and various other star formation tracers in order to investigate the nature of the dust heating sources in this spectral domain. The analysis is carried out using a sample of 123 normal, late-type, nearby galaxies with available data at several frequencies. The mid-IR luminosity (normalized to the $H$-band luminosity) correlates better with the far-IR luminosity than with more direct tracers of the young stellar population such as the $\mathrm{H} \alpha$ and the UV luminosity. The comparison of resolved images reveals a remarkable similarity in the $\mathrm{H} \alpha$ and mid-IR morphologies, with prominent HII regions at both frequencies. The mid-IR images, however, show in addition a diffuse emission not associated with HII regions nor with the diffuse $\mathrm{H} \alpha$ emission. This evidence indicates that the stellar population responsible for the heating of dust emitting in the mid-IR is similar to that heating big grains emitting in the far-IR, including relatively evolved stars responsible for the non-ionizing radiation. The scatter in the mid-IR vs. H $\alpha$, UV and far-IR luminosity relation is mostly due to metallicity effects, with metal-poor objects having a lower mid-IR emission per unit star formation rate than metal-rich galaxies. Our analysis indicates that the mid-IR luminosity is not an optimal star formation tracer in normal, late-type galaxies.
\end{abstract}

Key words. galaxies: spiral - galaxies: ISM - stars: formation - infrared: ISM

\section{Introduction}

The atomic gas, a major constituent of proto-galaxies, collapsed into molecular clouds to form stars at a rate variable with time. At least a fraction of elliptical galaxies was formed during a violent, rapid $\left(\leq 10^{8} \mathrm{yr}\right)$ collapse of the primordial gas, efficiently transformed into stars, probably induced by merging events, frequent in the dense early Universe. In spiral discs the rotation prevented a rapid collapse of the primordial gas, which formed a reservoir able to sustain the star formation up to the present epoch. The current star formation activity of nearby spiral galaxies seems primarily governed by the gas surface density, possibly modulated by the galactic differential rotation or by the stellar surface density (Kennicutt 1998a; Boissier et al. 2003). The star formation history of these objects should thus have been monotonic, governed by the physical properties of the original protogalaxies such as their angular momentum (Sandage 1986) or total mass (Boselli et al. 2001).

The study of the evolution of galaxies requires some reconstruction of their star formation activity from their birth to the present. In the nearby Universe the present day star formation activity of normal, late-type galaxies can be easily determined by combining $\mathrm{H} \alpha$ or UV data with population synthesis models (Kennicutt 1998a; Boselli et al. 2001). The major limitation in this technique is the determination of the dust extinction. Low-resolution integrated spectroscopy in the visible domain, now available for large galaxy samples (Kennicutt 1992; Jansen et al. 2000; Gavazzi et al. 2004), as well as far-IR data from the IRAS survey, however, allow us to accurately quantify the amount of extinction using the Balmer decrement or the far-IR to UV flux ratio (Buat et al. 2002).

Quantifying the star formation activity of galaxies at higher redshift is a more complex task. For $z \geq 0.5$ the $\mathrm{H} \alpha$ line shifts outside the optical domain, entering the less accessible near-IR part of the spectrum, where the atmospheric opacity and the sky instability make the determination of the $\mathrm{H} \alpha$ line intensity and of the Balmer decrement more difficult. Deep optical observations provide the UV rest-frame photometry, but the determination of the dust extinction, necessary to transform the 
UV flux into a quantitative star formation rate, can be accomplished only when far-IR data are known. These will soon be available for small patches of the sky from the SWIRE-Spitzer survey $\left(\sim 70 \mathrm{deg}^{2}\right)$. Since at redshifts higher than $z \sim 1$ most of the star formation should manifest itself by a major starburst inside the galaxy (Steidel et al. 1999), where extinction is particularly high, Balmer line and UV luminosities are not reliable star formation indicators. As shown by Kennicutt (1998a,b), for these highly extincted starburst galaxies the far-IR emission becomes the best star formation indicator. Far-IR data presently available from IRAS, ISO, Spitzer and shortly from ASTRO-F are however limited by the poor spatial resolution, of the order of $1.5-0.5^{\prime}$, making confusion a major limitation.

Mid-IR data have been proposed as a promising alternative star formation tracer, particularly suitable for highly extincted environments because less affected by obscuration than other indicators at shorter wavelength (Roussel et al. 2001; Förster Schreiber et al. 2004). Compared to far-IR, the mid-IR data have the advantage of a superior spatial resolution $(\sim$ a factor of 10 depending on wavelength). Furthermore, the mid-IR emission of late-type galaxies is expected to be more tightly related to the young stellar population than the far-IR one (Désert et al. 1990) thus representing a potentially more direct star formation tracer. In quiescent spiral galaxies, indeed, the far-IR emission is known to depend also on the old stellar population via circumstellar and/or photospheric emission or diffuse cirrus emission (Sauvage \& Thuan 1994).

The one to one relationship between mid-IR emission and recent star formation, was however questioned by Boselli et al. (1997a, 1998) who found a lack of correlation between the mid-IR emission at 6.75 and $15 \mu \mathrm{m}$ and various star formation tracers, and concluded that the carriers of the UIBs responsible for the emission at these wavelengths might be destroyed in high UV radiation fields, as first suggested by Boulanger et al. (1988) and Helou et al. (1991).

To understand the reason for this evident discrepancy, it is important to investigate in depth, both empirically and theoretically, the relationship between the mid-IR emission and the star formation rate. The emission of the ISM in the mid-IR is due to very small grains (VSG; $10 \AA \lesssim a \lesssim 200 \AA$ ) responsible for the continuum, and to smaller particles, not in thermal equilibrium with the radiation, responsible for the Unidentified Infrared Bands (UIBs). The mid-IR emission can also be due to $\mathrm{H}_{2}$ rotational lines, to fine-structure lines of various metals and $\mathrm{H}$ recombination lines produced in HII and photodissociation regions (Sturm et al. 2000) (the latter contribute only to a small fraction of the flux in the ISOCAM bands centered at 6.75 and $15 \mu \mathrm{m}$ and can be neglected). The small grains are stochastically heated to very high temperatures by the absorption of individual photons. UIBs are supposedly associated to polycyclic aromatic hydrocarbon molecules (PAHs, Léger \& Puget 1984; Désert et al. 1990) or to hydrogenated amorphous carbon grains (Duley \& Williams 1981, 1988). The study of the mid-IR spectral energy distribution (SED) and of its relationships with the UV radiation field in various extragalactic (IR bright galaxies, active galactic nuclei (AGN), normal late-type galaxies, blue compact dwarf galaxies (BCDs), ellipticals) and galactic environments (HII regions, diffuse medium, reflection nebulae...) (see for a review Genzel \& Cesarsky 2000) was recently boosted by the ISO and COBE missions. Spectroscopic studies showed that UIBs dominate the spectrum in the range 6-13 $\mu \mathrm{m}$ of normal (Lu et al. 2003) and starburst (Förster Schreiber et al. 2003) galaxies, as well as in Galactic HII and photodissociation regions (Roelfsema et al. 1996; Cesarsky et al. 1996; Verstraete et al. 1996, 2001), planetary and reflection nebulae (Beintema et al. 1996; Boulanger et al. 1996; Uchida et al. 1998) and in the diffuse medium associated to dust cirrus (Mattila et al. 1996; Lemke et al. 1998; Chan et al. 2001). VSGs dominate the emission at longer wavelengths but also in the $6-13 \mu \mathrm{m}$ regime whenever the UV radiation field is extremely high and/or the metallicity is low, as in BCDs and Wolf-Rayet galaxies (Madden 2000; Galliano et al. 2003; Crowther et al. 1999), AGN (Roche et al. 1991; Dudley 1999; Laurent et al. 2000), or close to prominent HII regions in low metallicity environments, such as N66 in the SMC (Contursi et al. 2000).

Further compelling evidence for the lack of direct relation between mid-IR dust emission and star formation comes from detailed studies of the physical properties of the ISM in the Milky Way and in some nearby galaxies. These studies showed that the presence of UIBs in the mid-IR spectrum is not necessarily associated to strong UV radiation, indicating that the carriers of the UIBs can be effectively excited also by less energetic photons produced by relatively evolved stars (Uchida et al. 1998; Pagani et al. 1999).

The complex behavior of the different components of the ISM observed in our Galaxy or in a few well studied nearby objects has made it possible to realize how difficult it is to interpret the global emission of unresolved galaxies observed with broad band filters, thus without detailed spectral information, when all the various components of the ISM (HII regions, cirrus, photo-dissociation regions (PDRs), diffuse medium) are mixed together and the properties of the interstellar radiation field change from object to object.

With this aim in mind, e.g. to better quantify if the mid-IR emission is a reliable star formation tracer in late-type galaxies, we propose in this work a re-discussion, based on a multifrequency statistical approach, of the nature of the dust heating sources responsible for the mid-IR emission in normal galaxies in conjunction with the properties of the UV radiation field and with the metallicity of the ISM. The major improvement with respect to our previous analysis (Boselli et al. 1997a, 1998) on this issue lies in the use of a better and larger mid-IR data-set homogeneously reduced using the latest ISOCAM data reduction pipeline and the availability of better imaging and spectroscopic material.

This work is the natural continuation of earlier statistical analyses (Boselli et al. 1997a, 1998) based on a large sample of late-type galaxies in the Virgo cluster that were observed by the ISOCAM consortium in guaranteed time and on several objects in the Coma supercluster region (Contursi et al. 2001). A revised version of this data set was presented in Boselli et al. (2003a) and was discussed by Boselli et al. (2003b) who reconstructed the UV-to-centimetric spectral energy distribution (SED) of normal, late-type galaxies. In summary, the analysis completed so far indicated that the system mass rather than 
the morphological type regulates the mid-IR emission (Boselli et al. 1998) and that in late-type normal galaxies the contribution of the cold stellar component to the total mid-IR emission is relevant, becoming dominant in quiescent, early-type spirals.

\section{The sample}

The study of the mid-IR properties of galaxies is mostly limited by the lack of data for homogeneous, complete and large samples of galaxies in the nearby Universe. The present work is based on the analysis of the sample of galaxies in the Virgo cluster and in the Coma/A1367 supercluster region by Boselli et al. (2003a). This sample, which includes 145 objects with homogeneously reduced ISOCAM 6.75 and $15 \mu \mathrm{m}$ imaging data of which 123 are late-type objects, is not complete. However, as extensively discussed in Boselli et al. (2003a,b), it includes an optically selected, volume limited, complete sample of 100 late-type galaxies in the Virgo cluster. The following analysis will be limited to the 123 late-type galaxies of type SaIm-BCD in the luminosity range $-21 \leq M_{B} \leq-13$.

\section{The data}

Mid-IR 6.75 ( $L W 2)$ and $15 \mu \mathrm{m}(L W 3)$ ISOCAM imaging data have been taken from Boselli et al. (2003a). To avoid large uncertainties in the mid-IR data, we considered as detections only those with quality 1 and 2 in Table 2 of Boselli et al. (2003a). As discussed in Boselli et al. (2003a), it is difficult to estimate the effective uncertainty in the mid-IR fluxes; here we assume a conservative $30 \%$.

Mid-IR luminosities at 6.75 and $15 \mu \mathrm{m}$ (in solar units) are estimated as in Boselli et al. (1998):

$L_{6.75 \mu \mathrm{m}}=4 \pi D^{2} F_{6.75 \mu \mathrm{m}} 2.724 \times 10^{2} L_{\odot}$

and

$L_{15 \mu \mathrm{m}}=4 \pi D^{2} F_{15 \mu \mathrm{m}} 1.184 \times 10^{2} L_{\odot}$

where $D$ is the distance in Mpc, and $F_{6.75 \mu \mathrm{m}}$ and $F_{15 \mu \mathrm{m}}$ are the mid-IR flux densities in mJy (for a bandwidth of $\delta_{6.75 \mu \mathrm{m}}=$ $11.57 \times 10^{12} \mathrm{~Hz}$ and $\delta_{15 \mu \mathrm{m}}=5.04 \times 10^{12} \mathrm{~Hz}$ respectively) .

Three different tracers are used to estimate the star formation activity of the target galaxies: the $\mathrm{H} \alpha$, the UV and the far-IR luminosity. $\mathrm{H} \alpha+[\mathrm{NII}]$ imaging and/or aperture photometry data, available for 96 galaxies, are taken from Boselli et al. (2002a, 2003b), Boselli \& Gavazzi (2002), Gavazzi et al. (1991, 1998, 2002b), Iglesias-Paramo et al. (2002), Kennicutt \& Kent (1983), Kennicutt et al. (1984) and references therein ${ }^{1}$.

Spectroscopic data, necessary to correct $\mathrm{H} \alpha$ imaging data for [NII] contamination and dust extinction taken from the survey of Gavazzi et al. (2004), are available for 91 objects. Long slit, drift-scan mode spectra were obtained by drifting the slit over the whole galaxy disc, as in Kennicutt (1992), thus providing values representative of the whole object. These are

\footnotetext{
${ }^{1}$ Fluxes from Kennicutt \& Kent (1983) data are increased by $16 \%$ as specified in Kennicutt et al. (1994) (also Kennicutt private communication) to account for a telluric line contamination in the off band filter.
}

intermediate $(\lambda / \Delta \lambda \sim 1000)$ resolution spectra in the range (3600-7200 ̊).

$\mathrm{H} \alpha$ imaging and aperture photometry data were corrected for dust extinction using the Balmer decrement given in Gavazzi et al. (2004), determined for 78 out of the 91 galaxies using available spectroscopic data. This correction is quite accurate since it takes into account the contribution of the underlying Balmer absorption to $\mathrm{H} \beta$. For those objects without integrated spectroscopy or measured Balmer decrement (20\%) we applied the corrections proposed by Boselli et al. (2002b) for the $[\mathrm{NII}]$ contamination $\left(\log ([\mathrm{NII}] / \mathrm{H} \alpha)=0.35 \times \log L_{\mathrm{H}}-\right.$ 3.85), and Boselli et al. (2001) for extinction: $A(\mathrm{H} \alpha)=1.1 \mathrm{mag}$ for Sa-Scd and Pec galaxies, and $A(\mathrm{H} \alpha)=0.6$ mag for the other objects. Consistently with Iglesias-Paramo et al. (2004) we also assume that the fraction of ionized photons absorbed by dust is $\sim 40 \%$, leading to a fraction of ionizing photons absorbed by the gas $f=0.6$. The mean error on the $\mathrm{H} \alpha$ luminosity is $\sim 15 \%$.

The spectroscopic survey of Gavazzi et al. (2004) was also used to estimate metallicities as $12+\log (\mathrm{O} / \mathrm{H})$, available for 68 objects. Metallicities have been estimated by averaging various calibrations, namely van Zee et al. (1998), Kewley \& Dopita (2002), Kobulnicky et al. (1999), McGaugh (1991), Dutil \& Roy (1999).

The UV data are taken from observations with FAUST (Deharveng et al. 1994, at $1650 \AA$ ) and FOCA (Donas et al. 1991,1995 , at $2000 \AA$ ). As in Boselli et al. (2003b) to be consistent with the FOCA data we transform FAUST UV magnitudes taken at $1650 \AA$ to $2000 \AA$ assuming a constant color index $\mathrm{UV}(2000)=\mathrm{UV}(1650)+0.2 \mathrm{mag}$. UV magnitudes used in the following analysis thus refer to a wavelength of $2000 \AA$. These are total magnitudes, determined by integrating the UV emission up to the weakest detectable isophote. The average estimated error in the UV magnitude is $0.3 \mathrm{mag}$, but it ranges from $0.2 \mathrm{mag}$ for bright galaxies to $0.5 \mathrm{mag}$ for weak sources observed in frames with larger than average calibration uncertainties. UV data are corrected for dust attenuation as described in Boselli et al. (2003b): $82 \%$ of the 62 galaxies detected at 60 and $100 \mu \mathrm{m}$ by IRAS or ISOPHOT have UV data: for these objects the UV extinction is determined from the FIR to UV flux ratio using the calibration given in Boselli et al. (2003b). Objects undetected at 60 or $100 \mu \mathrm{m}$ were corrected using the average UV extinction determined for galaxies of similar morphological type: $A(\mathrm{UV})=1.28 ; 0.85 ; 0.68 \mathrm{mag}$ for Sa-Sbc; Sc-Scd; Sd-Im-BCD galaxies respectively.

Far-IR IRAS fluxes at 60 and $100 \mu \mathrm{m}$ have been taken from different sources. Alternative ISOPHOT far-IR values at 60 and $100 \mu \mathrm{m}$ have been taken from Tuffs et al. (2002), bringing to 62 the number of objects with available far-IR data. The comparison of ISO and IRAS data for the sample galaxies detected in both surveys reveals a systematic difference of ISO/IRAS $=0.95$ and 0.82 at 60 and $100 \mu \mathrm{m}$ respectively (Tuffs et al. 2002). To be consistent with IRAS, ISOPHOT data have been multiplied by these factors. Far-IR luminosities are determined from the 60 and $100 \mu \mathrm{m}$ flux densities as in Boselli et al. (2003b). The mean uncertainty in the far-IR data is $15 \%$.

An accurate determination of the $\mathrm{H} \alpha$, UV and far-IR uncertainties determined with a similar set of data is given in Iglesias-Paramo et al. (2004). 
To compare galaxies of different sizes we remove the well known luminosity-luminosity or luminosity-mass scaling relations by normalizing luminosities in all bands to the $H$ band luminosity, which is a good tracer of the total dynamical mass of late-type galaxies (Gavazzi et al. 1996a). Near-IR data have been taken from our $H$ and $K^{\prime}$ band surveys of the Virgo cluster and of the Coma/A1367 supercluster (Boselli et al. 1997b, 2000; Gavazzi et al 1996b,c, 2000a,b). Total $H$ band luminosities have been determined using the relation: $\log L(H)=$ $11.36-0.4 \times H_{\mathrm{T}}+2 \log D$, where $H_{\mathrm{T}}$ is the total $H$ band magnitude and $D$ is the distance in Mpc. Total extrapolated nearIR magnitudes have been determined as described in Gavazzi et al. (2000a), with a mean uncertainty of $\sim 10 \%$. $K^{\prime}$ magnitudes have been transformed into $\mathrm{H}$ magnitudes adopting a constant $H-K^{\prime}=0.25$ (independent of type; see Gavazzi et al. 2000a) when the color index is not available. This is an additional source of uncertainty.

We recall that the quoted uncertaintes are mean values: the uncertainties in the spectro-photometric quantities of individual galaxies are larger and represent a source of dispersion in the relationships analysed throughout this work.

As shown by Boselli et al. (1998, 2003b), the emission of late-type galaxies in the mid-IR is partly contaminated by stellar emission. At $6.75 \mu \mathrm{m}$ the emission of the photosphere of the cold stellar population is dominant in Sa galaxies, where it reaches on average $\sim 80 \%$ of the total emission, while its contribution decreases in later types: in $\mathrm{Sc}$ it is on average $\sim 20 \%$, and $\sim 50 \%$ in BCDs.

As proposed by Boselli et al. (2003b), the stellar contribution at $6.75 \mu \mathrm{m}$ can be estimated from the SED determined by fitting the UV to near-IR spectrophotometric data with the updated version of Bruzual \& Charlot population synthesis models (Bruzual \& Charlot 1993; GISSEL 2001). Owing to the available multifrequency dataset, this can be achieved for most (75) of the galaxies. For the remaining (48) objects the pure dust emission has been estimated by subtracting from the $6.75 \mu \mathrm{m}$ emission the average stellar contribution for a given morphological type, as determined from the template SED given in Boselli et al. (2003b). We remind the reader that the evolutionary synthesis models used in this work to estimate the integrated mid-IR emission of late-type galaxies rely on either empirical stellar libraries and model atmospheres that are poorly known for cool stars, especially for evolved post-mainsequence stars dominating the galaxy emission at this wavelength. Another source of error of the fitting procedure is associated to the uncertainty in the star formation history and metallicity assumed for the model.

The accuracy of the morphological classification is excellent for the Virgo galaxies (Binggeli et al. 1985, 1993). Because of the larger distances, the morphology of galaxies belonging to the other surveyed regions suffers from an uncertainty of about 1.5 Hubble type bins. We assume a distance of $17 \mathrm{Mpc}$ for the members (and possible members) of Virgo cluster A, $22 \mathrm{Mpc}$ for Virgo cluster B, and $32 \mathrm{Mpc}$ for objects in the M and W clouds (see Gavazzi et al. 1999). Members of the clusters Coma and A1367 are assumed to be at distances of 86.6 and $92 \mathrm{Mpc}$ respectively. Isolated galaxies in the Coma supercluster are assumed to be at their redshift
Table 1. The number of objects with available data in various bands and the completeness with respect to the 123 late-type galaxies considered in this work.

\begin{tabular}{lc}
\hline \hline Data & No. of objects (\%) \\
\hline H $\alpha$ & $96(78 \%)$ \\
UV & $57(46 \%)$ \\
FIR (detected) & $62(50 \%)$ \\
NIR & $114(93 \%)$ \\
Spectroscopy & $91(74 \%)$ \\
Balmer dec. & $78(63 \%)$ \\
Metallicity & $68(55 \%)$ \\
SED for stellar correction & $75(61 \%)$ \\
\hline
\end{tabular}

distance adopting $H_{0}=75 \mathrm{~km} \mathrm{~s}^{-1} \mathrm{Mpc}^{-1}$. Most of the data used in this analysis are available in the GOLDMine database (http//goldmine.mib.infn.it; Gavazzi et al. 2003).

\section{Analysis}

\subsection{Star formation tracers}

The multifrequency dataset outlined above is well suited for studying the nature and the relative contribution of the different heating sources of the dust emitting in the mid-IR. Three different tracers of star formation, the $\mathrm{H} \alpha$, UV and FIR luminosities, can be determined from the available data.

The $\mathrm{H} \alpha$ luminosity gives a direct measure of the global photoionization rate of the interstellar medium due to highmass $\left(m>10 M_{\odot}\right)$, young $\left(\leq 10^{7}\right.$ years) O-B stars (Kennicutt 1983; Kennicutt et al. 1994; Boselli et al. 2001). If the SFR is constant over a time scale of some $10^{7}$ years (the life time of the ionizing stars), $\mathrm{H} \alpha$ luminosity can be converted into SFR (in $M_{\odot} \mathrm{yr}^{-1}$ ) assuming an initial mass function and using population synthesis models, as extensively described in Boselli et al. (2001).

The UV emission of galaxies at $2000 \AA$ is dominated by less young $\left(\sim 10^{8}\right.$ years $)$ and massive $\left(2<m<5 M_{\odot}\right) \mathrm{A}$ stars (Lequeux 1988). If the SFR is stationary on time scales $\geq 3 \times 10^{8}$ years $^{2}$, as for the $\mathrm{H} \alpha$, UV luminosities can be converted into SFR using population synthesis models. These two tracers are thus independent. The major limitation of both indicators, however, is dust attenuation. Although both tracers are accurately corrected using totally independent recipes (the extinction in $\mathrm{H} \alpha$ is determined from the Balmer decrement and in the UV from the far-IR to UV flux ratio), the SFR determined from these two indicators can suffer from similar biases and limitations.

The far-IR emission due to the dust heated by the stellar radiation field, although not directly tracing the emission of the young stellar population, has the advantage of being extinction-free. As extensively discussed in the literature (Kennicutt 1998b; Sauvage \& Thuan 1994, and references therein), the far-IR emission is an accurate star formation tracer only in strong starburst galaxies, where most of the stellar

\footnotetext{
2 This assumption is reasonable for normal galaxies like those analyzed in this work (Boselli et al. 2001; Gavazzi et al. 2002a).
} 

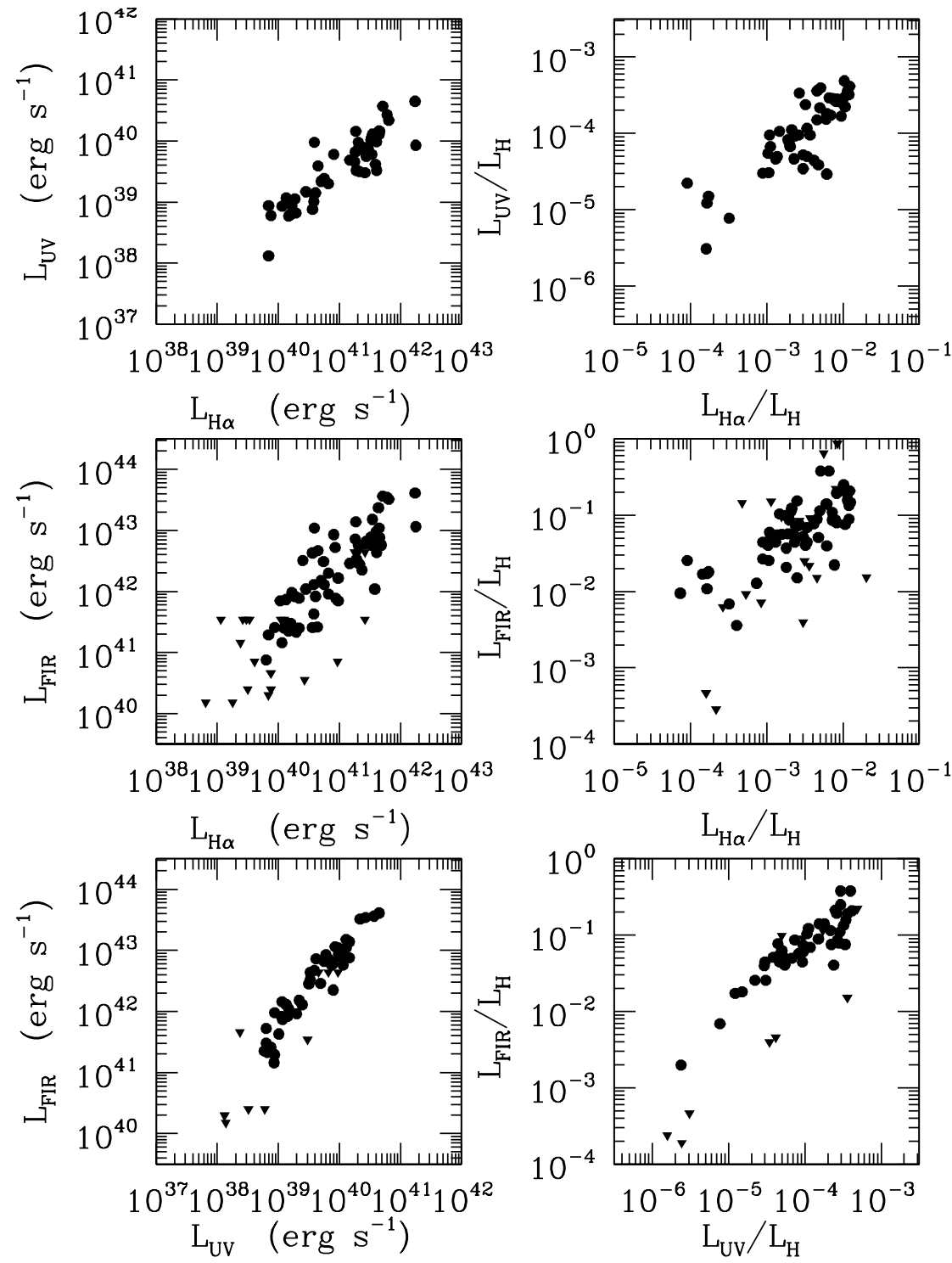

Fig. 1. The relationship between the $\mathrm{H} \alpha$, the UV and the far-IR luminosities (left panels) and normalized to the near-IR luminosity (right panel). Triangles indicate upper limits to the far-IR luminosity.

radiation, particularly that emitted by the young, massive stars, is absorbed by dust and re-radiated in the IR. In normal galaxies like those analyzed in this work, however, the contribution of the dust heating by the radiation emitted by the older stellar population can be significant, in particular in the most quiescent objects (Sauvage \& Thuan 1994).

The correlations between the three tracers are shown in Fig. 1. As previously mentioned, the strong relationship between the $\mathrm{H} \alpha$, UV and far-IR luminosities (left panels) is primarily a scaling effect (bigger galaxies have more of everything). For this reason the same relationships are also given normalized to the near-IR luminosity in the right column. Note that the dispersions in the UV vs. $\mathrm{H} \alpha$ and FIR vs. UV relationships are significantly smaller than that in the FIR vs. $\mathrm{H} \alpha$ relationships. While the small dispersion in the UV vs. $\mathrm{H} \alpha$ luminosity relationship is expected because both quantities trace the young stellar population and suffer from similar extinction effects ${ }^{3}$, that between the FIR and the UV luminosity might reflect the fact that UV and FIR luminosities are not entirely independent entities, because FIR data have been used to correct the UV luminosity for dust attenuation.

\subsection{The origin of the mid-IR emission in late-type galaxies}

\subsubsection{Integrated values}

To study the origin of the mid-IR emission of normal, late-type galaxies we compare in Fig. 2 the normalized 6.75 and $15 \mu \mathrm{m}$

${ }^{3}$ The dispersion in the UV vs. $\mathrm{H} \alpha$ luminosity relationship is probably partly due to uncertainties in the dust extinction correction and partly due to recent star formation events (Iglesias-Paramo et al. 2004). 


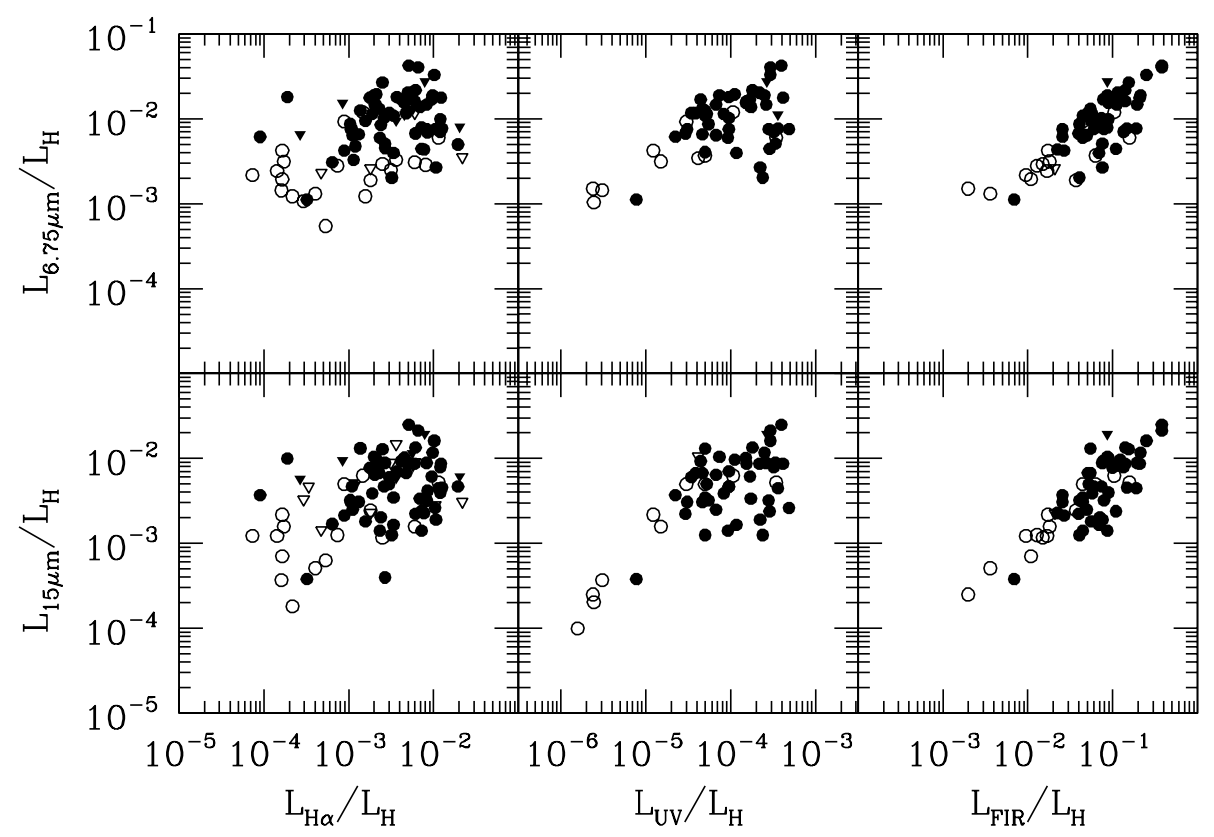

Fig. 2. The relationship between the normalized (to the $H$ band) mid-IR luminosities at 6.75 (upper) and $15 \mu \mathrm{m}$ (lower) and the H $\alpha$, the UV and the far-IR luminosity. Filled symbols are for those objects whose mid-IR emission is dominated by dust $\left(L_{\mathrm{D}}+L_{\mathrm{S}} / L_{\mathrm{S}}(6.75 \mu \mathrm{m})>2\right)$, open symbols for galaxies whose mid-IR emission is mostly stellar $\left(L_{\mathrm{D}}+L_{\mathrm{S}} / L_{\mathrm{S}}(6.75 \mu \mathrm{m}) \leq 2\right)$, which we consider the most doubtful data $\left(L_{\mathrm{D}}\right.$ stands for dust luminosity, $L_{\mathrm{S}}$ for stellar luminosity). Triangles indicate upper limits to the mid-IR luminosity.

luminosity with that of the three star formation tracers previously analyzed.

Figure 2 shows a general correlation between the mid-IR emission (in both bands) and the three star formation tracers. The dispersion is however small only for the correlation with the far-IR luminosity. If we limit the comparison to objects whose mid-IR emission is dominated by dust (filled symbols in Fig. 2), avoiding a large unwanted stellar contamination, the dynamical range in the mid-IR luminosity is significantly reduced, the correlation with the $\mathrm{H} \alpha$ strongly weakens, and that with the UV luminosity almost vanishes.

We thus need to correct the mid-IR emission for the stellar contribution. This correction can be achieved at $6.75 \mu \mathrm{m}$, since the stellar emission can be estimated from the Bruzual \& Charlot models fitted to the stellar SEDs (Boselli et al. 2003b), see Sect. 3. Throughout Figs. 2, 3, 6, 9, and 10 we keep separate the galaxies with mid-IR emission dominated by dust $\left(L_{\mathrm{D}^{+}}\right.$ $L_{\mathrm{S}} / L_{\mathrm{S}}(6.75 \mu \mathrm{m})>2$ (filled symbols), requiring minor corrections to remove the stellar contribution, from objects whose emission is almost entirely stellar $\left(L_{\mathrm{D}}+L_{\mathrm{S}} / L_{\mathrm{S}}(6.75 \mu \mathrm{m}) \leq 2\right)$, so that their mid-IR dust emission is highly uncertain (empty symbols); in the above $L_{\mathrm{D}}$ stands for pure dust and $L_{\mathrm{S}}$ for stellar luminosity. A similar correction cannot be applied at $15 \mu \mathrm{m}$ since the Bruzual \& Charlot models are limited to $\lambda \leq 10 \mu \mathrm{m}$ : the stellar contamination at wavelengths larger than $10 \mu \mathrm{m}$ should however be minor.

The pure dust luminosity at $6.75 \mu \mathrm{m}, L_{\mathrm{D} 6.75 \mu \mathrm{m}}$ (normalized to the total $H$ band luminosity), vs. the three star formation tracers is plotted in Fig. 3. There is still a general correlation between the mid-IR dust emission and the far-IR emissivity, although weaker than when uncorrected data are used, but the correlations with UV and $\mathrm{H} \alpha$, if any, have become extremely weak.

These trends however rely on the most doubtful $6.75 \mu \mathrm{m}$ measurements (empty symbols in Fig. 3).

It is remarkable that the dispersion in the $L(6.75 \mu \mathrm{m}) / L(H)$ vs. $L(\mathrm{H} \alpha) / L(H)$ relation is larger than in the $L(\mathrm{FIR}) / L(H)$ vs. $L(\mathrm{H} \alpha) / L(H)$ relation (Fig. 1). This suggests that the dispersion in the $L(6.75 \mu \mathrm{m}) / L(H)$ vs. $L(\mathrm{H} \alpha) / L(H)$ relation is independent of the extinction because errors in the extinction correction would introduce a similar scatter in both relations.

\subsubsection{Resolved galaxies}

We further investigate the origin of the mid-IR emission in late-type galaxies by comparing the 2-D 6.75 (and $15 \mu \mathrm{m}$ images) of galaxies resolved by ISOCAM with the images tracing the distribution of the young stellar population. Because of the poor ISOCAM spatial resolution $\left(6^{\prime \prime}\right.$ at $\left.6.75 \mu \mathrm{m}\right)$, the number of fully resolved objects showing some structure (nucleus, spiral arms, compact regions) is limited to a dozen galaxies. Images tracing the distribution of the young stars refer at present to the $\mathrm{H} \alpha+[\mathrm{NII}]$ images (available for all the sample galaxies). UV images will shortly be available from GALEX, while the far-IR images from IRAS do not have enough resolution $\left(\sim 1.5^{\prime}\right)$ for a meaningful comparison.

Once again mid-IR images must be corrected for the stellar contamination. The $6.75 \mu \mathrm{m}$ emission of some of the resolved galaxies of the sample of Boselli et al. (2003a) is in fact totally stellar, as it is not associated to any recent star formation event. As an example the elliptical galaxy NGC 4649, companion of the spiral NGC 4647, has an extended emission in the mid-IR, but no recent star formation activity, as shown in Fig. 4. As in 


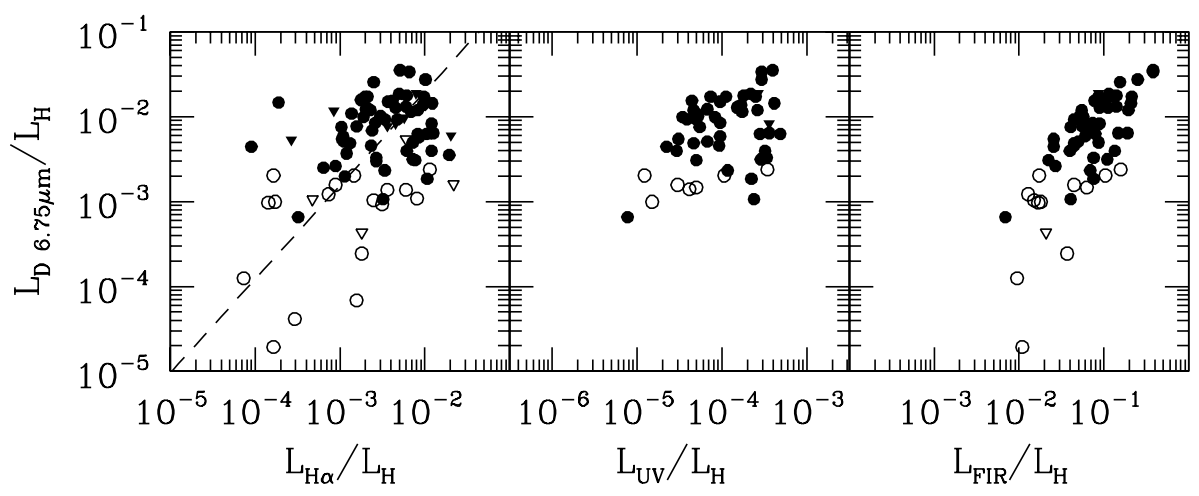

Fig. 3. The relationship between the normalized (to the $H$ band) mid-IR luminosity at 6.75 corrected for the stellar contribution and the H $\alpha$, the UV and the far-IR luminosity. Symbols as in Fig. 2.

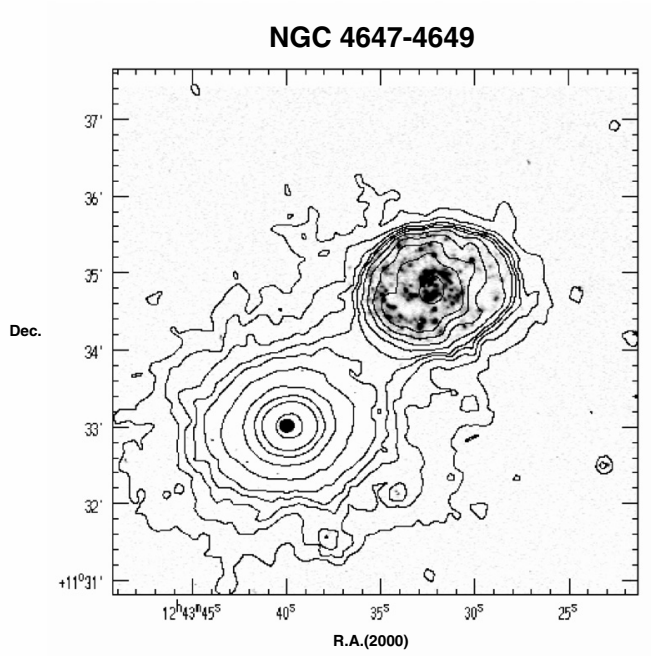

Fig. 4. The contour plot of the $6.75 \mu \mathrm{m}$ emission (dust and stars) superimposed on the grey scale $\mathrm{H} \alpha+[\mathrm{NII}]$ net image of the galaxies NGC 4647 and 4649. Contours are given at 2, 4, 6, 8, 10, 15, 25, $40,60,150,300 \mu \mathrm{Jy} \operatorname{arcsec}^{-2}$. The grey scale is in arbitrary units.

Boselli et al. (2003b), we reconstruct the pure $6.75 \mu \mathrm{m}$ emitting dust distribution by assuming that the 2-D distribution of stars emitting at $\sim 7 \mu \mathrm{m}$ is similar to that at $\sim 2 \mu \mathrm{m}$. Thus we subtract from the $6.75 \mu \mathrm{m}$ images those in the $K^{\prime}$ band (appropriately scaled in flux using the fitted UV-to-near-IR SED and smoothed to the same resolution ${ }^{4}$ ). As previously remarked, this correction cannot be applied to the $15 \mu \mathrm{m}$ images: at these wavelengths, however, we can consider that the stellar contribution is only marginal. The contour plots of the $6.75 \mu \mathrm{m}$ dust emission (uncontaminated by stars) of the resolved galaxies are superimposed on the grey scale $\mathrm{H} \alpha+[\mathrm{NII}]^{5}$ net images

\footnotetext{
${ }^{4}$ We thus make the reasonable assumption that, on large scales, the effect due to an extinction gradient can be neglected.

5 As for the integrated values, to trace star formation $\mathrm{H} \alpha+[\mathrm{NII}] \mathrm{im}-$ ages should be corrected for dust extinction and [NII] contribution. Although a radial variation of both entities is expected (Boissier et al. 2004), this should be minor, thus affecting the star forming morphology of these galaxies only marginally.
}

in Fig. $5^{6}$. Despite the difference in spatial resolution ( $66^{\prime \prime}$ for ISOCAM vs. $\sim 1-2.5^{\prime \prime}$ for $\mathrm{H} \alpha$ ), the ionizing flux and the mid-IR images show a remarkably similar morphology. All bright spots resolved at $6.75 \mu \mathrm{m}$ are clearly associated with star forming HII regions. In addition, however, the ISOCAM images show a low-surface-brightness diffuse emission extending over the optical disc of the galaxies not associated with star forming $\mathrm{HII}$ regions nor with diffuse $\mathrm{H} \alpha$ emission. Although radiative transfer processes cannot be totally excluded, in relatively quiescent galaxies such as the Milky Way (see next section) the disc interstellar radiation field outside HII regions is not necessarily produced by the youngest stellar population, but by stars showing a range of ages and masses (Uchida et al. 1998; Pagani et al. 1999). This suggests that PAHs and VSG, dominating the emission of galaxies in the mid-IR, are mostly heated by the young stellar population inside HII regions, while they are heated by all stellar populations all throughout their discs.

\section{Discussion}

The analysis carried out so far on both resolved and unresolved galaxies led us to the conclusion that the carriers of the UIB and associated continuum responsible for the integrated mid-IR emission of normal, late-type galaxies are heated by both the UV ionizing and non-ionizing radiation emitted by the young and old stellar populations. This evidence indicates that the dust emitting in the mid-IR is heated in the same way as the big grains emitting in the far-IR for which the contribution of visible and near-IR photons produced by low-mass old stars is well established. This result agrees with what is found in the Milky Way and in M 31, where UIBs have been detected in the diffuse ISM in regions with low UV radiation (Sellgren et al. 1990; Mattila et al. 1996; Uchida et al. 1998; Pagani et al. 1999; Li \& Draine 2002a).

It is difficult to know to what extent the contribution of the non-ionizing radiation differs in galaxies of different morphological type and/or luminosity. The emission of dust at $6.75 \mu \mathrm{m}$ is only weakly related to the morphological type, increasing from Sa to Sd, Im and peculiar galaxies, but decreasing again in BCDs, as shown in Fig. 6. This may be due to

\footnotetext{
${ }^{6}$ The $15 \mu \mathrm{m}$ images of the same galaxies, not given here, show morphologies similar to those at $6.75 \mu \mathrm{m}$.
} 
a lower abundance of dust in those metal-poor galaxies. The relationship with the total mass of galaxies is even more complex: the $L_{\mathrm{D} 6.75 \mu \mathrm{m}} / L_{H}$ ratio increases with the $H$ band luminosity from $L_{H}=10^{8}$ to $10^{10.5} L_{H \odot}$ (filled dots in Fig. 6), but might decrease at higher luminosities. It seems therefore that the mid-IR pure dust emission is more intense in massive, quiescent, metal-rich objects than in star forming, metal-poor dwarfs.

\subsection{Comparison with previous results}

Boselli et al. (1997a, 1998) observed an anti-correlation between the mid-IR dust emission and the UV to $H$ band flux ratio in the high star-formation regime. This behavior is not confirmed by the present analysis, being hidden in the high dispersion of the mid-IR vs. H $\alpha$ and vs. UV diagrams of Fig. 2. The mid- vs. far-IR relationship just shows a higher dispersion in the most active galaxies, while the different behavior among star forming and quiescent galaxies in the mid-IR vs. UV relationships is mostly due to objects whose mid-IR emission is dominated by stars (empty symbols). All these properties, however, disappear once the pure dust emission is used (Fig. 3).

This conclusion seems to be in contrast with the results of Boselli et al. (1998), where mid-IR data corrected for the stellar contribution were used, weakening (although not excluding) their conclusion that PAHs are destroyed in strong UV fields. This discrepancy with our 1998 work could be due to:

i) the new data reduction pipeline, incorporating a more accurate transient correction (see the detailed discussion in Boselli et al. 2003a) gives more reliable mid-IR fluxes in comparison with the data extracted using the old ISOCAM pipeline that suffer from a systematic overestimate of the mid-IR fluxes in low luminosity objects. Low luminosity galaxies are the most active star forming objects in our sample;

ii) UV data in Boselli et al. (1998) were not corrected for dust extinction.

The analysis carried out in the previous section has shown that the integrated dust emission of late-type galaxies in the mid-IR is not fully related to the intensity of the ionizing UV radiation field. This result is in agreement with our previous findings based on smaller and less complete samples (Boselli et al. 1997a, 1998), but contrasts with the conclusions of Roussel et al. (2001) and Förster Schreiber et al. (2004) who found a tight correlation between the 6.75 and $15 \mu \mathrm{m}$ and the $\mathrm{H} \alpha$ surface brightness in spiral discs. This apparent discrepancy can be due to several factors, that we wish to analyze:

1) Systematic sample differences.

The sample analyzed by Roussel et al. (2001) includes 49 galaxies, out of which 20 are Virgo members, in common with our work. Their sample, however, spans the luminosity range $-19.2 \geq M_{B} \geq-21.4$, and is thus dominated by massive galaxies, while our analysis is based on objects of lower average luminosity, with $-15 \geq M_{B} \geq-21.5$. The physical properties of the ISM are expected to change significantly with luminosity: although the average UV ionizing field of the two samples is similar (they both span the range $-2.3 \leq \log \Sigma \mathrm{H} \alpha \leq-0.2 L_{\odot} \mathrm{pc}^{-2}$ ), our sample includes low metallicity objects, with $8.2 \leq 12+\log (\mathrm{O} / \mathrm{H}) \leq$ 9.2. Metallicity measurements are not available for all the galaxies of Roussel et al. (2001), but given their luminosity we expect on average solar metallicities (Zaritsky et al. 1994). The sample of Förster Schreiber et al. (2004) contains the same spirals as Roussel et al. (2001) combined with active starburst and far-IR bright galaxies with $M_{B} \leq-19.2$ and $10^{42.6} \leq L_{\mathrm{FIR}} \leq 10^{45} \mathrm{erg} \mathrm{s}^{-1}$ and is thus still limited to solar metallicity objects.

2) Determination of the $\mathrm{H} \alpha$ luminosity.

$\mathrm{H} \alpha+[\mathrm{NII}]$ imaging data have been corrected for dust extinction and [NII] contamination using different recipes. While Roussel et al. (2001) (and thus Förster Schreiber et al. (2004) for the subsample of spiral discs) applied a constant correction both for the [NII] contamination $([\mathrm{NII}] / \mathrm{H} \alpha=0.33)$ and for extinction $(A(\mathrm{H} \alpha)=1.1 \mathrm{mag})$, our spectroscopic integrated data allowed a more accurate galaxy-by-galaxy correction. The use of constant corrections might have introduced systematic biases in the data. As shown by Gavazzi et al. (2004), the [NII]/H $\alpha$ ratio increases strongly with luminosity, ranging from $[\mathrm{NII}] / \mathrm{H} \alpha \sim$ 0.1 to $[\mathrm{NII}] / \mathrm{H} \alpha \sim 4$ when the luminosity increases from $M_{B}=-15$ to $M_{B}=-21.5$. In a similar way, the Balmer decrement is not constant, but changes significantly from galaxy to galaxy. Gavazzi et al. (2004) have shown that $A(\mathrm{H} \alpha)$ ranges between 0 and $\sim 5 \mathrm{mag}$ in late-type galaxies with higher extinctions in redder objects. We are thus confident that our $\mathrm{H} \alpha$ data are of higher quality than those for the disc galaxies of Roussel et al. (2001) ${ }^{7}$. It is however unclear why a constant correction of the $\mathrm{H} \alpha$ data should reduce the scatter in the mid-IR vs. $\mathrm{H} \alpha$ relations shown in these two works.

3) Normalization.

To compare galaxies of different size, mid-IR and $\mathrm{H} \alpha$ luminosities have been normalized by the optical area in Roussel et al. (2001) and Förster Schreiber et al. (2004) and by the $H$ band luminosity in this work. We prefer to use the dust free ${ }^{8} H$ band luminosity not only because it is a direct tracer of the total dynamical mass of normal, latetype galaxies (Gavazzi et al. 1996a), but also because it can be determined with a significantly higher accuracy $(\sim 10 \%)$ than the optical area, in particular in objects with an irregular morphology ( 30-50\%). However, as in point 2), we would expect that these more accurate corrections and normalizations would lower, instead of increase, the scatter of the mid-IR vs. H $\alpha$ relation.

4) Correction for stellar contribution.

Roussel et al. (2001) and Förster Schreiber et al. (2004) did not correct the mid-IR luminosities for the stellar contribution. While for far-IR and starburst galaxies this

\footnotetext{
${ }^{7}$ For several starburst galaxies the extinction given by Förster Schreiber et al. (2004) relies on near-IR hydrogen recombination lines.

${ }^{8}$ In dust-rich objects such as ultra luminous IR galaxies or starburst galaxies analysed in Förster Schreiber et al. (2004), extinction can be important even in the $H$ band.
} 

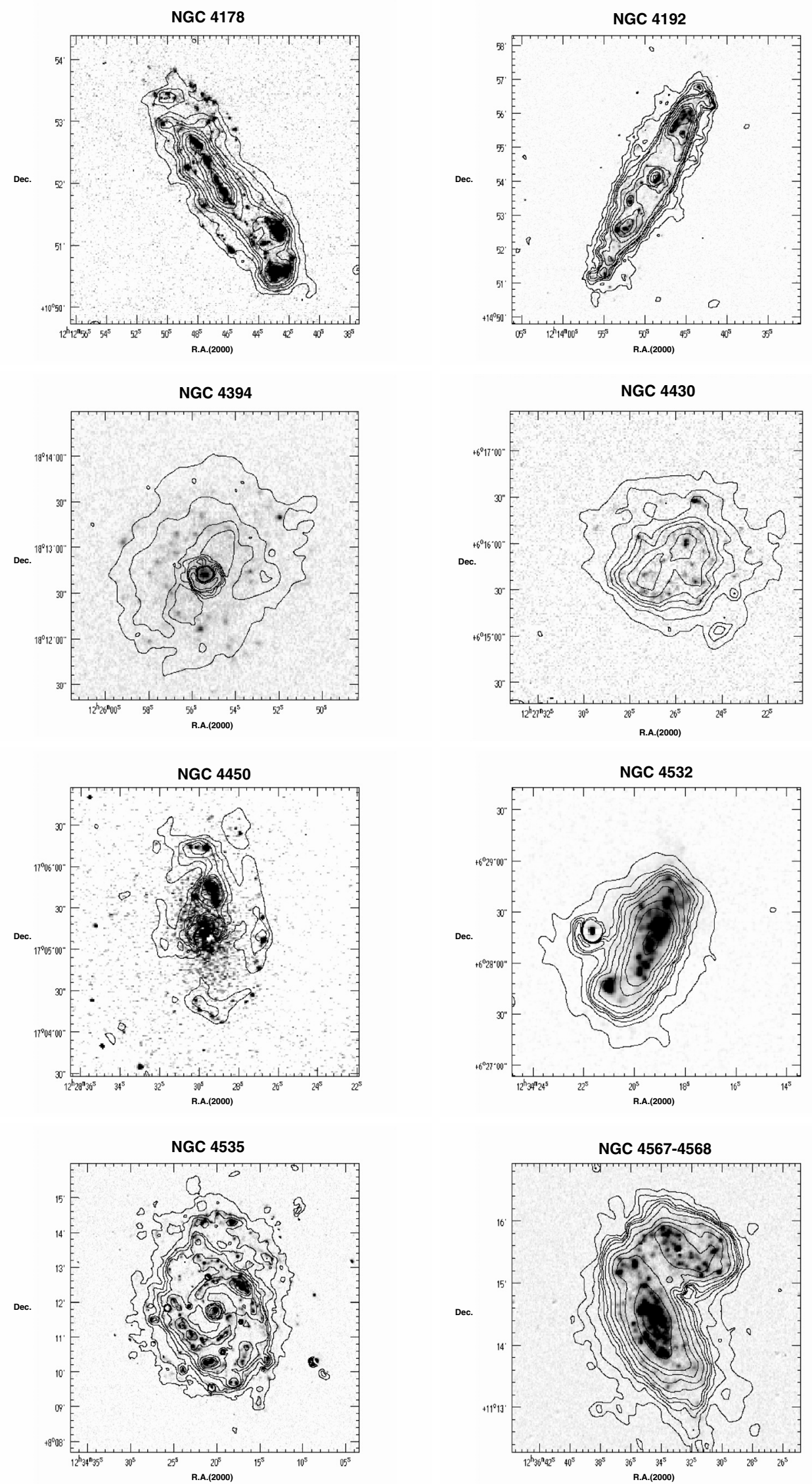

Fig. 5. The contour plot of the $6.75 \mu \mathrm{m}$ dust emission (without the contribution of the stellar emission) superimposed on the grey scale $\mathrm{H} \alpha+[\mathrm{NII}]$ net image of the resolved galaxies. Contours are given at 2, 4, 6, 8, 10, 15, 25, 40,60, 150, $300 \mu \mathrm{Jy} \operatorname{arcsec}^{-2}$. The grey scale is in arbitrary units. 

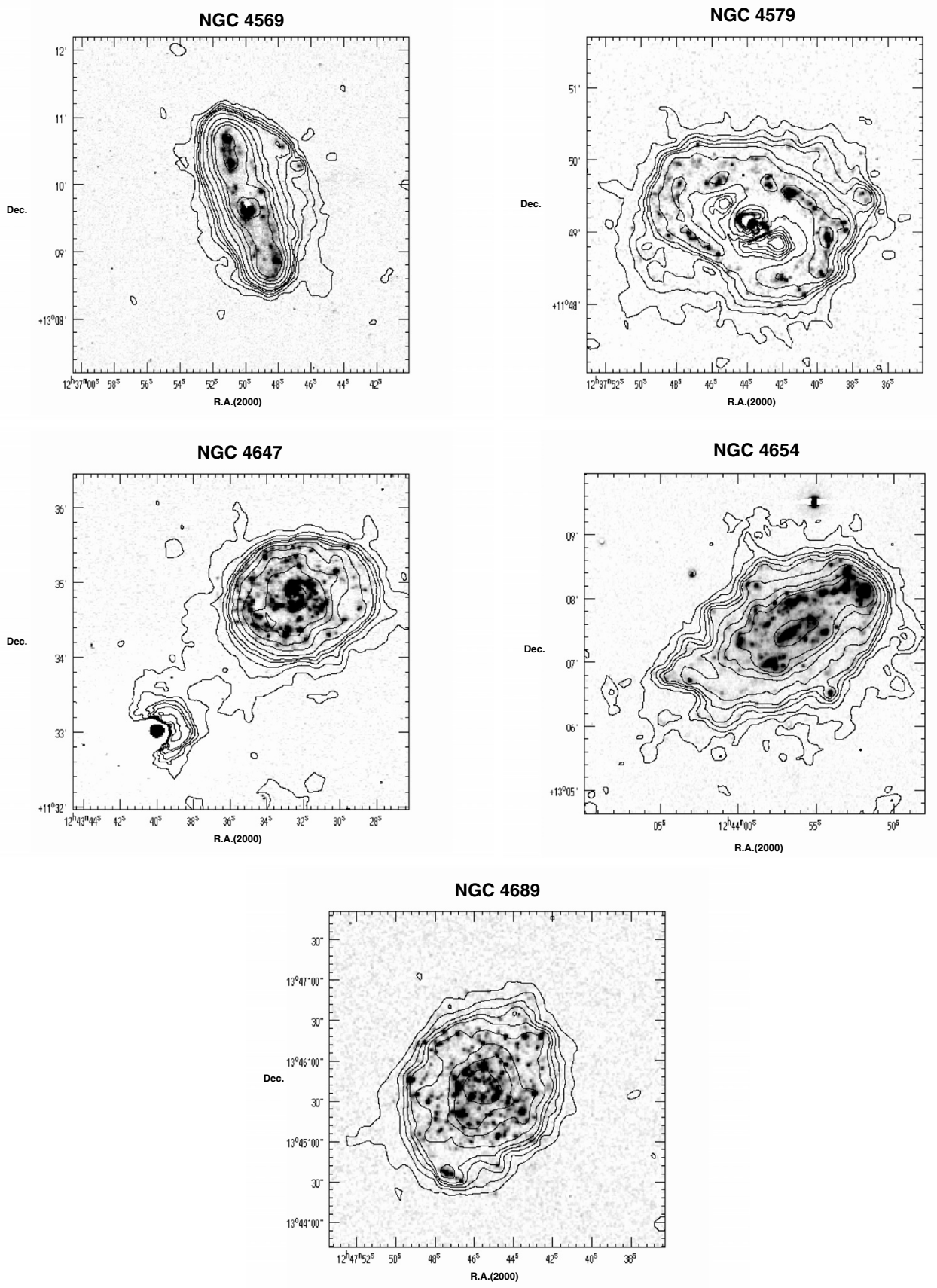

Fig. 5. continued.

contribution is probably negligible, in quiescent late-type galaxies $^{9}$ the stellar emission can be relevant particularly at $6.75 \mu \mathrm{m}$. A higher dispersion in our mid-IR vs. $\mathrm{H} \alpha$ relations with respect to those of Roussel et al. (2001) is however present also when uncorrected data are used (see Fig. 2).

5) Disc vs. nucleus.

To study the disc properties, Roussel et al. (2001) subtracted the contribution of the nucleus from both mid-IR and $\mathrm{H} \alpha$ data, decreasing the dispersion in the mid-IR vs.

\footnotetext{
${ }^{9}$ Seventeen out of the 49 galaxies in Roussel et al. (2001) (the Virgo galaxies) have a direct measure of the stellar contamination in the mid-IR in Boselli et al. (2003b).
}

$\mathrm{H} \alpha$ relation (see their Fig. 5). Because of the small angular extent of our sources we cannot apply a similar aperture correction; the stellar contamination correction that we use, however, should be to a first order equivalent since the stellar contribution to the mid-IR emission is dominant in the central part of late-type galaxies (Boselli et al. 2003a).

In conclusion we believe that the higher observed scatter in our mid-IR vs. H $\alpha$ (normalized) relation with respect to that observed by Roussel et al. (2001) and Förster Schreiber et al. (2004) is real and probably related to the diversity of the samples. 


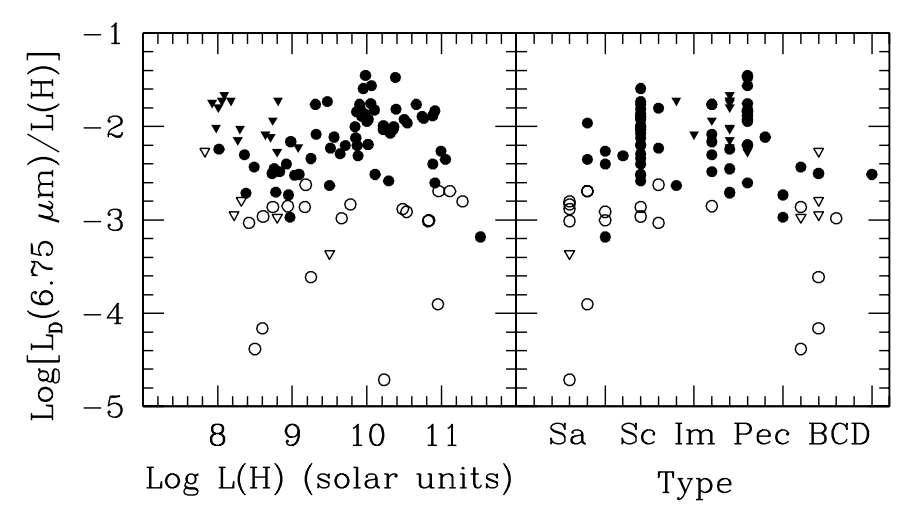

Fig. 6. The relationship between the normalized (to the $H$ band) dust emission mid-IR luminosity at $6.75 \mu \mathrm{m}$ and the $H$ band luminosity and the morphological type. Symbols as in Fig. 2.

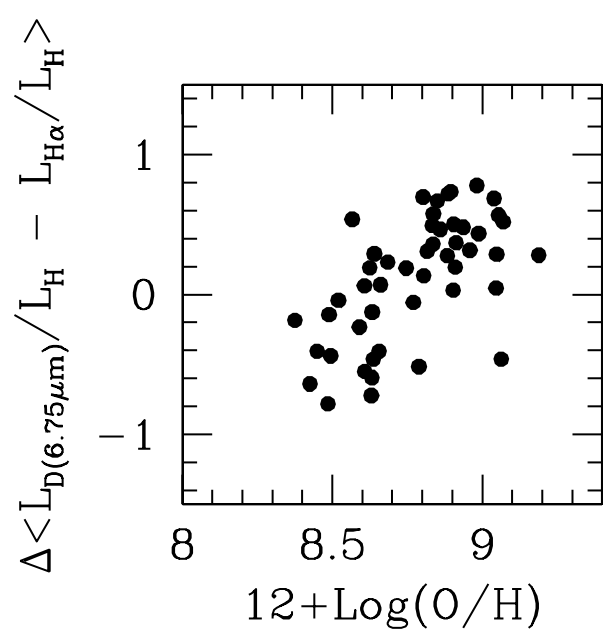

Fig. 7. The relationship between the residual of the pure dust $L_{\mathrm{D} 6.75 \mu \mathrm{m}}$ vs. $\mathrm{H} \alpha$ normalized luminosities, (on logarithmic scales) and the metallicity $12+\log \mathrm{O} / \mathrm{H}$ for detected galaxies with a mid-IR dust dominated emission.

\subsection{The origin of the scatter in the mid-IR vs. SFR relations}

To further investigate the origin of the scatter in the mid-IR vs. SFR relationships, we look for any systematic trend between the residual of the mid-IR pure dust $6.75 \mu \mathrm{m}$ vs. $\mathrm{H} \alpha$ normalized relationship, $\left(\log L_{\mathrm{D} 6.75 \mu \mathrm{m}} / L_{H}=1.105 \times \log L_{\mathrm{H} \alpha} / L_{H}+0.504\right)$, and any other parameters tracing the physical properties of the target galaxies. To rule out environmental effects, which in fact are not expected since the dust heating in the mid-IR is localized well inside the unperturbed optical disc of the galaxy, we checked that the residual of the mid-IR vs. $\mathrm{H} \alpha$ relationship $\left(\Delta\left\langle L_{\mathrm{D} 6.75 \mu \mathrm{m}} / L_{H}-L_{\mathrm{H} \alpha} / L_{H}\right\rangle\right)$ is independent of the HIdeficiency parameter ${ }^{10}$.

The residual of the mid-IR vs. $\mathrm{H} \alpha$ relationship is instead found to depend on the metallicity of the target galaxies, as shown in Fig. 7. In spite of the large dispersion, Fig. 7 shows

10 The HI-deficiency parameter is defined as the ratio of the HI mass to the average $\mathrm{HI}$ mass of isolated objects of similar morphological type and linear size (Haynes \& Giovanelli 1984), which is a good quantitative indicator of the galaxy-cluster ongoing interaction. that metal rich galaxies $(12+\log (\mathrm{O} / \mathrm{H}) \sim 9)$ have, on average, $\sim 10$ higher $6.75 \mu \mathrm{m}$ mid-IR dust emission per unit ionizing $\mathrm{UV}$ photons than metal poor objects $(12+\log (\mathrm{O} / \mathrm{H}) \sim 8.4)$. The higher dispersion in our mid-IR vs. $\mathrm{H} \alpha$ relation with respect to that of Roussel et al. (2001) and Förster Schreiber et al. (2004) can be ascribed to this effect since the mid-IR spectral properties of late-type galaxies are known to change with metallicity and our sample spans a broader metallicity range (see Fig. 8). As summarized by Madden (2000), the intensity of the UIBs in the 5-20 $\mu \mathrm{m}$ regime and their relative contribution with respect to the underlying continuum ${ }^{11}$ decrease in low metallicity environments, as observed in BCD galaxies (Thuan et al. 1999; Galliano et al. 2003), in Wolf-Rayet galaxies (Crowther et al. 1999), and in compact HII regions in the Magellanic Clouds (Contursi et al. 2000). In the Small Magellanic Cloud the UIBs are weak (Li \& Draine 2002b) with the exception of one quiescent cloud (Reach et al. 2000). Strong UIBs are instead present in metal rich environments with strong UV radiation fields like those encountered in luminous starburst galaxies (M 82, NGC 253, NGC 1808: Förster Schreiber et al. 2003), although their relative contribution to the mid-IR emission might decrease in extremely active systems (Lu et al. 2003). This can be interpreted by a lower abundance of PAHs due to the lower metallicity, or by their higher destruction by the UV field which propagates more easily due to the lower dust content, or by differences in the properties of the UIB carriers (see for the high-metallicity galaxy M 31, Cesarsky et al. 1998). The 6.75 to $15 \mu \mathrm{m}$ broad band filter ratio can be used to trace, though only qualitatively, the relative importance of the UIB to VSG emission since, as previously discussed, the ISOCAM LW3 $15 \mu \mathrm{m}$ filter encompasses both UIB features and dust continuum, while the LW2 $6.75 \mu \mathrm{m}$ filter is generally dominated by UIB emission.

Figure 9 does not show any evident trend of the mid-IR flux ratio with the intensity of the UV ionizing field (as traced by the $\mathrm{H} \alpha$ luminosity) or the metallicity (see also Fig. 1c in Roussel et al. 2001). We should remember, however, that the dynamic ranges of both the UV radiation field and metallicity sampled with our data are relatively small, preventing the determination of strong trends. Systematic variations in the UIB to VSG contribution to the mid-IR emission of galaxies because of depletion of the UIB carriers are in fact expected for radiation fields $\sim 10^{3.5}$ higher than in the solar neighborhood (Förster Schreiber et al. 2004), observed only in extremely metal poor objects such as IIZw40 $(12+\log (\mathrm{O} / \mathrm{H})=8.10 ;$ Diaz \& Perez-Montero 2000), NGC $1569(12+\log (\mathrm{O} / \mathrm{H})=8.19 ;$ Kobulnichky \& Skillman 1997) or SBS0335-052 $(12+\log (\mathrm{O} / \mathrm{H})=7.33$; Izotov et al. 1997) but not in NGC 1140, where the metallicity is only $12+\log (\mathrm{O} / \mathrm{H})=8.46$ (Guseva et al. 2000) (Madden 2000).

\footnotetext{
11 We recall that, as discussed in Förster Schreiber et al. (2004), the emission of normal galaxies in the $6.75 \mu \mathrm{m} L W 2$ ISO filter is generally dominated by the UIBs at $6.2,7.7$ and $8.6 \mu \mathrm{m}$, while both UIBs (the strong $12.7 \mu \mathrm{m}$ line and minor features at $13.55,14.25$ and $15.7 \mu \mathrm{m}$, probably dominating in low star formation regimes) and the underlying continuum due to VSGs contribute to the emission in the $15 \mu \mathrm{m}$ $L W 2$ ISO filter.
} 


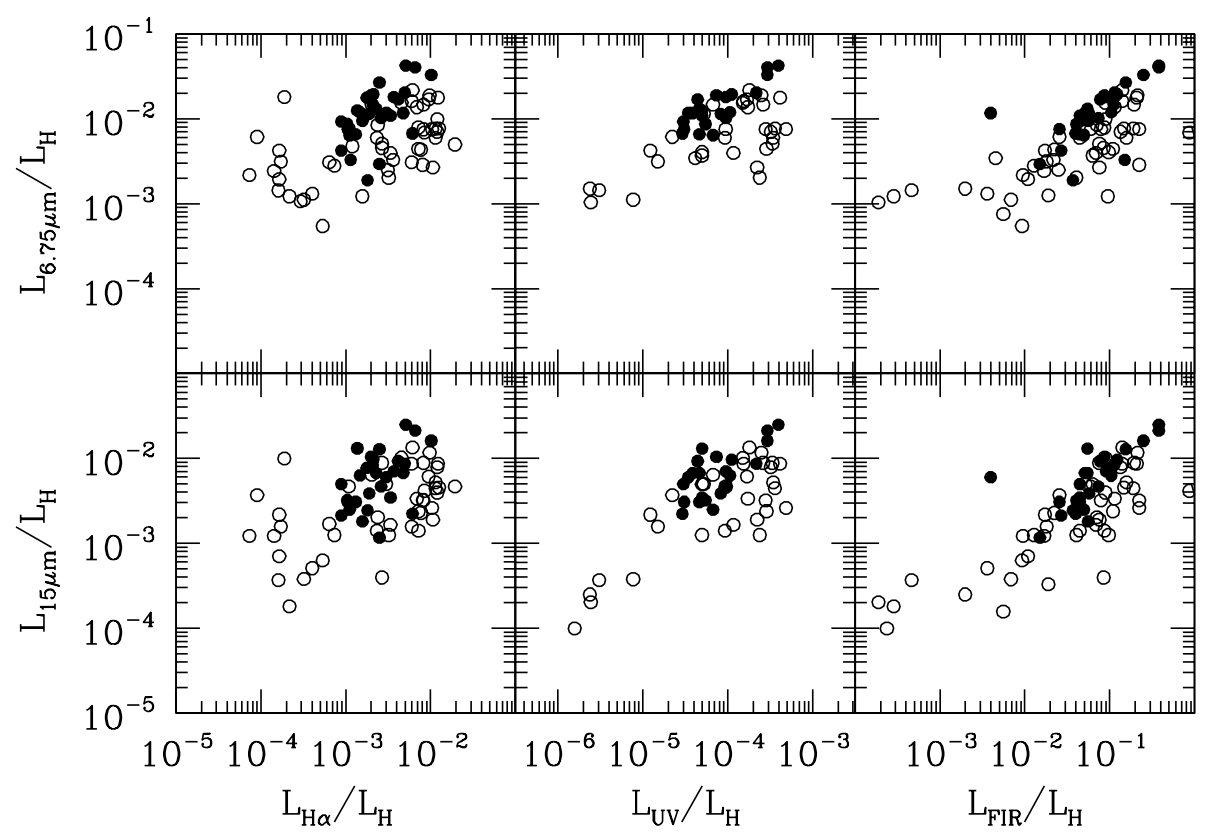

Fig. 8. The relationship between the normalized (to the $H$ band) mid-IR luminosities at 6.75 (upper) and $15 \mu \mathrm{m}$ (lower) and the H $\alpha$, the UV and the far-IR luminosity for detected galaxies. Filled dots are for galaxies with solar metallicities $(12+\mathrm{Log} \mathrm{O} / \mathrm{H}>8.8)$, empty symbols for sub-solar values $(12+\log \mathrm{O} / \mathrm{H} \leq 8.8)$

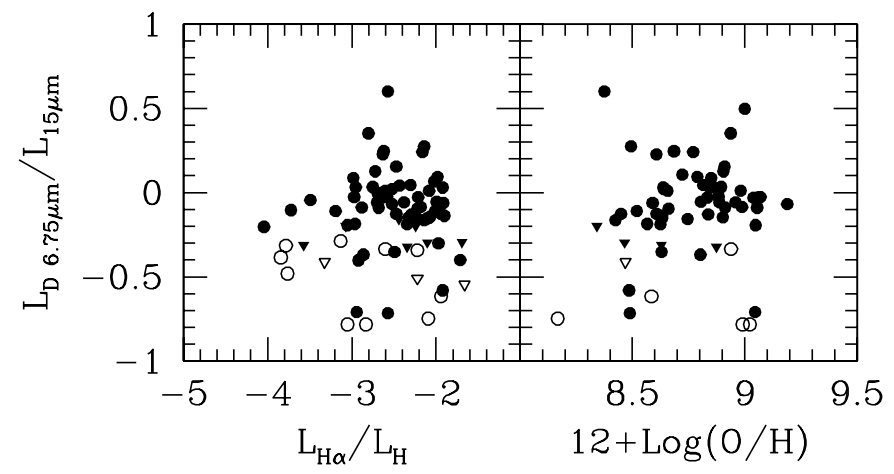

Fig. 9. The relationship of the mid-IR color $6.75 / 15 \mu \mathrm{m}$ flux ratio with the star formation activity (left) and with the metallicity (right). Symbols as in Fig. 2.

\subsection{The mid-IR emission as a star formation tracer}

The observational evidence discussed in the previous sections brings us to the conclusion that, despite different physical properties of the emitting dust, in normal galaxies it is roughly the same stellar population that is responsible for the heating of the dust emitting both in the mid- and far-IR. Since this population includes stars of different ages and masses, the first consequence is that, for these quiescent objects, the mid-IR emission is not a good tracer of the star formation. However, since the mid-IR emission is often used in the literature to measure the star formation, we try here to quantify the uncertainty introduced by this method.

A calibration based on radiative transfer models at these wavelengths, such as that used by Kennicutt (1998b) in the
far-IR, is not suited, not only because of the poorly known physical properties of the emitting dust, but also because dust is stochastically heated and not in thermal equilibrium with the radiation. We thus derive an empirical calibration by comparing the mid-IR luminosity to the luminosity in other bands that traditionally are assumed as star formation tracers, as in Boselli et al. (2002b). Since star formation estimates from mid-IR data generally apply to mid-IR selected samples, for which accurate stellar SED are unavailable, in this case we use total (uncorrected for stellar contamination) mid-IR luminosities. Figure 10 shows the relationship between the mid-IR 6.75 and $15 \mu \mathrm{m}$ luminosities and the $\mathrm{H} \alpha$, the UV and the far-IR luminosities for galaxies in our sample. As in Fig. 1, the strong relationship is just a scaling effect (none of the variables is normalized). We want to stress that some of the relationships are non-linear, as can be seen from the lack of a direct relation with star formation, and that they are all affected by a large dispersion, particularly when the mid-IR luminosity is plotted vs. the $\mathrm{H} \alpha$ and UV luminosities, confirming the large uncertainty of the calibration. The best fit to the data, obtained from a bisector linear regression is given in Table 2.

In the assumption that the star formation activity of these galaxies has been constant over the last $\sim 10^{7}$ years for $\mathrm{H} \alpha$, $\sim 3 \times 10^{8}$ years for $\mathrm{UV}$, the star formation rate of a galaxy (in solar masses per year) can be estimated from the relationship:

$S F R_{\lambda}=K_{\lambda} \times L_{\lambda} \quad M_{\odot} \mathrm{yr}^{-1}$.

Different calibration constants $K_{\lambda}$ valid for for several IMFs and metallicities can be found in the literature (Kennicutt 1998b; Boselli et al. 2001; Iglesias-Paramo et al. 2004), or can be directly estimated from population synthesis models. 


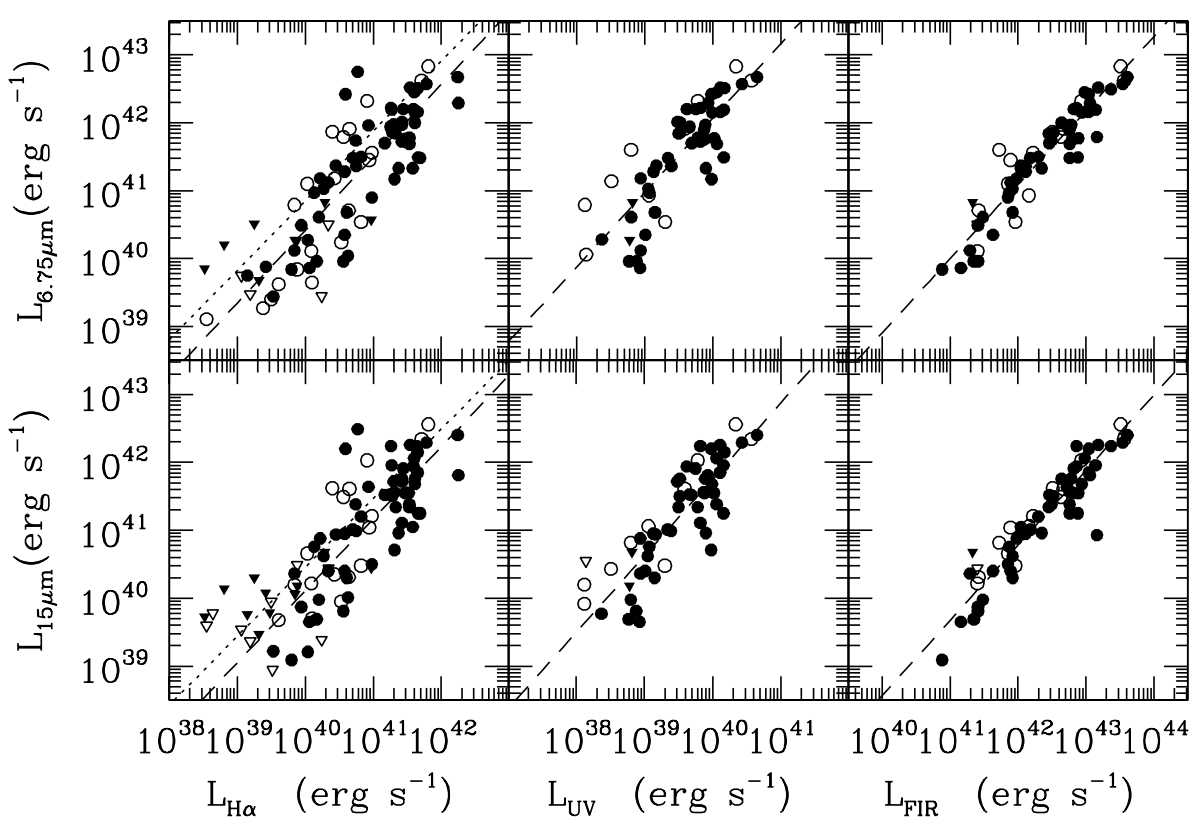

Fig. 10. The relationship between the mid-IR luminosities at 6.75 (upper) and $15 \mu \mathrm{m}$ (lower) and the $\mathrm{H} \alpha$, the UV and the far-IR luminosity. Filled symbols are for those objects whose mid-IR emission is dominated by dust $\left(L_{\mathrm{D}}+L_{\mathrm{S}} / L_{\mathrm{S}}(6.75 \mu \mathrm{m})>2\right)$, open symbols for galaxies whose mid-IR emission is mostly stellar $\left(L_{\mathrm{D}}+L_{\mathrm{S}} / L_{\mathrm{S}}(6.75 \mu \mathrm{m}) \leq 2\right)$. Triangles indicate upper limits to the mid-IR luminosities. The dashed line indicates the best fit given in Table 2, the dotted line the fit given in Roussel et al. (2001) corrected for the $f$ factor (see Sect. 3).

Table 2. Best fits to the relations between the mid-IR 6.75 and $15 \mu \mathrm{m}$ luminosities ( $x$ variable) and the three star formation tracers ( $y$ variable), with both variables on logarithmic scales.

\begin{tabular}{|c|c|c|c|c|}
\hline Variable & Slope & Constant & No. of objects & $R^{2 a}$ \\
\hline \multicolumn{5}{|c|}{$L(6.75 \mu \mathrm{m})$} \\
\hline$L(\mathrm{H} \alpha)$ & $0.788 \pm 0.040$ & $8.259 \pm 1.718$ & 81 & 0.71 \\
\hline$L(\mathrm{UV})$ & $0.757 \pm 0.051$ & $8.068 \pm 2.179$ & 54 & 0.68 \\
\hline$L(\mathrm{FIR})$ & $0.863 \pm 0.028$ & $6.568 \pm 1.258$ & 62 & 0.88 \\
\hline \multicolumn{5}{|c|}{$L(15 \mu \mathrm{m})$} \\
\hline$L(\mathrm{H} \alpha)$ & $0.751 \pm 0.049$ & $10.060 \pm 2.078$ & 74 & 0.62 \\
\hline$L(\mathrm{UV})$ & $0.750 \pm 0.045$ & $8.626 \pm 1.958$ & 54 & 0.73 \\
\hline$L(\mathrm{FIR})$ & $0.851 \pm 0.028$ & $7.322 \pm 1.240$ & 62 & 0.89 \\
\hline
\end{tabular}

${ }^{a}$ : regression coefficient.

For example, assuming a Salpeter IMF $(\alpha=2.35)$ in the mass range between 0.1 and $100 M_{\odot}$, Kennicutt (1998b) gives $K_{\mathrm{H} \alpha}=1 / 1.26 \times 10^{41}\left(M_{\odot} \mathrm{yr}^{-1} / \mathrm{erg} \mathrm{s}^{-1}\right)$, thus:

$S F R=1.441 \times 10^{-33} \times 10^{0.788 \times \log L_{6.75} \mu \mathrm{m}} \quad M_{\odot} \mathrm{yr}^{-1}$.

We stress that these calibrations apply to late-type galaxies in the mid-IR luminosity range between $10^{39} \leq L(6.75 \mu \mathrm{m}) \leq$ $10^{43} \mathrm{erg} \mathrm{s}^{-1}$ and $10^{39} \leq L(15 \mu \mathrm{m}) \leq 10^{42.6} \mathrm{erg} \mathrm{s}^{-1}$. We do not know whether these values apply outside this range, in particular at higher luminosities such as those encountered in far-IR bright galaxies. We also recall that, as extensively discussed in Kennicutt (1998b), far-IR luminosities can be transformed into SFR (in $M_{\odot} \mathrm{yr}^{-1}$ ) only in dusty starburst galaxies. Galaxies analyzed in this work have far-IR luminosities in the range $10^{41} \leq L(\mathrm{FIR}) \leq 10^{43.7} \mathrm{erg} \mathrm{s}^{-1}\left(10^{7.4} \leq L(\mathrm{FIR}) \leq 10^{10.1}\right.$ in solar units) and are normal, quiescent late-type galaxies. The use of the mid- vs. far-IR empirical calibration given in Table 2 would thus lead to highly uncertain results. The dispersion in the $L_{\mathrm{H} \alpha}$ vs. $L_{6.75 \mu \mathrm{m}}$ and $L_{15 \mu \mathrm{m}}$ relations is a factor of $\sim 5(1 \sigma)$. The uncertainty in the determination of the SFR of galaxies using Eq. (4) is still larger. As shown in Charlot \& Longhetti (2001), the uncertainty in the determination of SFR from $\mathrm{H} \alpha$ data using stellar population synthesis models is already a factor of $\sim 3$ when the data are properly corrected for dust extinction and $[\mathrm{NII}]$ contamination, as done in this work. If we take into account all the possible sources of error in the determination of the $\mathrm{H} \alpha$ luminosity, and the uncertainty introduced by the large dispersion observed in Fig. 10, we conclude that the resulting uncertainty in the determination of the SFR from mid-IR luminosity measurement is as high as a factor of $\sim 10$.

\section{Conclusion}

Using a sample of 123 normal, late-type, nearby galaxies with available multifrequency data we have studied the relationship between the mid-IR $(5-18 \mu \mathrm{m})$ emission and various other star formation tracers for investigating the nature of the dust heating sources in this spectral domain. This analysis has shown that the normalized mid-IR luminosity correlates better with the far-IR luminosity than with more direct tracers of the young stellar population such as the $\mathrm{H} \alpha$ and the UV luminosity. The comparison of resolved images reveals a remarkable similarity in the $\mathrm{H} \alpha$ and mid-IR morphology, with all HII regions prominent at both frequencies. However, once corrected for the stellar contamination, mid-IR images are also characterized by a diffuse emission not associated with HII regions nor with the diffuse $\mathrm{H} \alpha$ emission.

Although radiation transfer processes cannot be excluded, this evidence, in agreement with similar results obtained for the 
ISM of our own Galaxy, indicates that the stellar population responsible for the heating of dust emitting in the mid-IR is similar to that which heats big grains emitting in the far-IR. The dust emission of a whole galaxy in the 5-18 $\mu \mathrm{m}$ domain is thus due to dust heated by both the ionizing and non-ionizing radiation, the latter produced mainly by relatively evolved stars. The direct consequence of our analysis is that the mid-IR luminosity is not an optimal star formation tracer in normal, late-type galaxies. We further analyzed the origin of the scatter in the mid-IR vs. H $\alpha$, UV and far-IR luminosity relationships and demonstrated that mostly has to do with metallicity effects, with metal-poor objects having a lower mid-IR emission per unit star formation rate than metal-rich galaxies.

The present results hold for normal, late-type galaxies and do not necessarely apply to luminous starburst galaxies or ultra-luminous IR galaxies. These extreme objects are not very frequent in the nearby Universe but are probably representative of forming galaxies detected in mid-IR deep surveys. Förster Schreiber et al. (2004) claim that for actively-starforming galaxies the mid-IR luminosity is a good star formation tracer. This apparent discrepancy will be resolved once extensive spectro-photometric surveys of galactic and extragalactic sources in a variety of physical conditions will help to understand the connection between the mid-IR dust emission properties (namely the relative contribution of the UIB line and continuum emission, the destruction of the UIB carriers, the contribution of the very small grains, etc.) and the physical conditions of the ISM, namely the metallicity and the UV radiation field.

Acknowledgements. We would like to thank V. Buat, A. Contursi and J.M. Deharveng for interesting discussions, and L. Cortese for the determination of the best fits. We would like to thank the anonymous referee for his comments and suggestions which helped to improve the quality of the manuscript.

\section{References}

Beintema, D., van den Ancker, M. E., Molster, F. J., et al. 1996, A\&A, 315, L369

Binggeli, B., Sandage, A., \& Tammann, G. 1985, AJ, 90, 1681 (VCC)

Binggeli, B., Popescu, C., \& Tammann, G. 1993, A\&AS, 98, 275

Boissier, S., Prantzos, N., Boselli, A., \& Gavazzi, G. 2003, MNRAS, 346,1215

Boissier, S., Boselli, A., Buat, V., Donas, J., \& Milliard, B. 2004, A\&A, 424, 465

Boselli, A., \& Gavazzi, G. 2002, A\&A, 386, 124

Boselli, A., Lequeux, J., Contursi, A., et al. 1997a, A\&A, 324, L13

Boselli, A., Tuffs, R., Gavazzi, G., Hippelein, H., \& Pierini, D. 1997b, A\&A, 121, 507

Boselli, A., Lequeux, J., Sauvage, M., et al. 1998, A\&A, 335, 53

Boselli, A., Gavazzi, G., Franzetti, P., Pierini, D., \& Scodeggio, M. 2000, A\&AS, 142, 73

Boselli, A., Gavazzi, G., Donas, J., \& Scodeggio, M. 2001, AJ, 121, 753

Boselli, A., Iglesias-Paramo, J., Vilchez, J. M., \& Gavazzi, G. 2002a, A\&A, 386, 134

Boselli, A., Gavazzi, G., Lequeux, J., \& Pierini, D. 2002b, A\&A, 385, 454

Boselli, A., Sauvage, M., Lequeux, J., Donati, A., \& Gavazzi, G. 2003a, A\&A, 406, 867
Boselli, A., Gavazzi, G., \& Sanvito, G. 2003b, A\&A, 402, 37

Boulanger, F., Beichman, C., Désert, F., et al. 1988, ApJ, 332, 328

Boulanger, F., Reach, W. T., Abergel, A., et al. 1996, A\&A, 315, L325

Bruzual, G., \& Charlot, S. 1993, ApJ, 405, 538

Buat, V., Boselli, A., Gavazzi, G., \& Bonfanti, C. 2002, A\&A, 383, 801

Cesarsky, D., Lequeux, J., Abergel, A., et al. 1996, A\&A, 315, L309

Cesarsky, D., Lequeux, J., Pagani, L., et al. 1998, A\&A, 337, L35

Chan, K. W., Roellig, T. L., Onaka, T., et al. 2001, ApJ, 546, 273

Charlot, S., \& Longhetti, M. 2001, MNRAS, 323, 887

Contursi, A., Lequeux, J., Cesarsky, D., et al. 2000, A\&A, 362, 310

Contursi, A., Boselli, A., Gavazzi, G., et al. 2001, A\&A, 365, 11

Crowther, P., Beck, S. C., Willis, A., et al. 1999, MNRAS, 304, 654

Deharveng, J. M., Sasseen, T. P., Buat, V., et al. 1994, A\&A, 289, 715

Désert, X., Boulanger, F., \& Puget, J. L. 1990, A\&A, 237, 215

Diaz, A., \& Perez-Montero, E. 2000, MNRAS, 312, 130

Donas, J., Milliard, B., \& Laget, M. 1991, A\&A, 252, 487

Donas, J., Milliard, B., \& Laget, M. 1995, A\&A, 303, 661

Dudley, C. C. 1999, MNRAS, 307, 553

Duley, W., \& Williams, D. 1981, MNRAS, 196, 269

Duley, W., \& Williams, D. 1988, MNRAS, 230, 1

Dutil, Y., \& Roy, J. 1999, ApJ, 516, 62

Förster Schreiber, N., Sauvage, M., Charmandaris, V., et al. 2003, A\&A, 399, 833

Förster Schreiber, N., Roussel, H., Sauvage, M., \& Charmandaris, V. 2004, A\&A, 419, 501

Galliano, F., Madden, S., Jones, A., et al. 2003, A\&A, 407, 159

Gavazzi, G., Boselli, A., \& Kennicutt, R. 1991, AJ, 101, 1207

Gavazzi, G., Pierini, D., \& Boselli, A. 1996a, A\&A, 312, 397

Gavazzi, G., Pierini, D., Boselli, A., \& Tuffs, R. 1996b, A\&AS, 120, 489

Gavazzi, G., Pierini, D., Baffa, C., et al. 1996c, A\&AS, 120, 521

Gavazzi, G., Catinella, B., Carrasco, L., Boselli, A., \& Contursi, A. 1998, AJ, 115, 1745

Gavazzi, G., Boselli, A., Scodeggio, M., Belsole, E., \& Pierini, D. 1999, MNRAS, 304, 595

Gavazzi, G., Franzetti, P., Scodeggio, M., Boselli, A., \& Pierini, D. 2000a, A\&A, 361, 863

Gavazzi, G., Franzetti, P., Scodeggio, M., et al. 2000b, A\&AS, 142, 65

Gavazzi, G., Bonfanti, C., Sanvito, G., Boselli, A., \& Scodeggio, M. 2002a, ApJ, 576, 135

Gavazzi, G., Boselli, A., Pedotti, P., Gallazzi, A., \& Carrasco, L. 2002b, A\&A, 386, 114

Gavazzi, G., Boselli, A., Donati, A., Franzetti, P., \& Scodeggio, M. 2003, A\&A, 400, 451

Gavazzi, G., Zaccardo, A., Sanvito, G., Boselli, A., \& Bonfanti, C. 2004, A\&A, 417, 499

Genzel, R., \& Cesarsky, C. 2000, ARA\&A, 38, 761

Guseva, N., Izotov, Y., \& Thuan, T. 2000, ApJ, 531, 776

Haynes, M., \& Giovanelli, R. 1984, AJ, 89, 758

Helou, G., Ryter, C., \& Soifer, B. 1991, ApJ, 376, 505

Jansen, R., Fabricant, D., Franx, M., \& Caldwell, N. 2000, ApJS, 126, 331

Iglesias-Paramo, J., Boselli, A., Cortese, L., Vilchez, J. M., \& Gavazzi, G. 2002, A\&A, 384, 383

Iglesias-Paramo, J., Boselli, A., Gavazzi, G., \& Zaccardo, A. 2004, A\&A, 421, 887

Izotov, Y. I., Lipovetsky, V. A., Chaffee, F. H., et al. 1997, ApJ, 476, 698

Kennicutt, R. 1983, ApJ, 272, 54

Kennicutt, R. 1998a, ARA\&A, 36, 189

Kennicutt, R. 1998b, ApJ, 498, 541 
Kennicutt, R. 1992, ApJ, 388, 310

Kennicutt, R., \& Kent, S. 1983, AJ, 88, 1094

Kennicutt, R., Bothun, G., \& Schommer, R. 1984, AJ, 89, 1279

Kennicutt, R., Tamblyn, P., \& Congdon, C. 1994, ApJ, 435, 22

Kewley, L. J., \& Dopita, M. A. 2002, ApJS, 142, 35

Kobulnicky, H. A., \& Skillman, E. D. 1997, ApJ, 489, 636

Kobulnicky, H. A., Kennicutt, R., \& Pizagno, J. 1999, ApJ, 514, 544

Laurent, O., Mirabel, I. F., Charmandaris, V., et al. 2000, A\&A, 359, 887

Léger, A., \& Puget, J. L. 1984, A\&A, 137, L5

Lemke, D., Mattila, K., Lehtinen, K., et al. 1998, A\&A, 331, 742

Lequeux, J. 1988, in Lecture Notes in Physics: Evolution of Galaxies; Astronomical Observations, EADN Astrophysics School I, Les Houches, France, ed. I. Appenzeller, H. Habing, \& P. Léna, 147

Li, A., \& Draine, B. 2002a, ApJ, 572, 232

Li, A., \& Draine, B. 2002b, ApJ, 576, 762

Lu, N., Helou, G., Werner, M., et al. 2003, ApJ, 588, 199

Madden, S. 2000, NewAR, 44, 249

Mattila, K., Lemke, D., Haikala, L., et al. 1996, A\&A, 315, L353

McGaugh, S. 1991, ApJ, 380, 140

Pagani, L., Lequeux, J., Cesarsky, D., et al. 1999, A\&A, 351, 447
Reach, W., Boulanger, F., Contursi, A., \& Lequeux, J. 2000, A\&A, 361, 895

Roche, P., Aitken, D., Smith, C. H., \& Ward, M. 1991, MNRAS, 248, 606

Roelfsema, P., Cox, P., Tielens, A., et al. 1996, A\&A, 315, L289

Roussel, H., Sauvage, M., Vigroux, L., \& Bosma, A. 2001, A\&A, 372, 427

Sandage, A. 1986, A\&A, 161, 89

Sauvage, M., \& Thuan, T. 1994, ApJ, 429, 153

Sellgren, K., Luan, L., \& Werner, M. 1990, ApJ, 359, 384

Steidel, C., Adelberger, K., Giavalisco, M., Dickinson, M., \& Pettini, M. 1999, ApJ, 519, 1

Sturm, E., Lutz, D., Tran, D., et al. 2000, A\&A, 358, 481

Thuan, T., Sauvage, M., \& Madden, S. 1999, ApJ, 516, 783

Tuffs, R. J., Popescu, C., Pierini, D., et al. 2002, ApJS, 140, 609

Uchida, K., Sellgren, K., \& Werner, M. 1998, ApJ, 493, L109

van Zee, L., Salzer, J., Haynes, M., O'Donoghue, A., \& Balonek, T. 1998, AJ, 116, 2805

Verstraete, L., Puget, J. L., Falgarone, E., et al. 1996, A\&A, 315, L337

Verstraete, L., Pech, C., Moutou, C., et al. 2001, A\&A, 372, 981

Zaritsky, D., Kennicutt, R., \& Huchra, J. 1994, ApJ, 420, 87 